\title{
A Novel Algorithm for Automatic Species Identification Using Principal Component Analysis
}

\author{
Shreyas Sen, Seetharam Narasimhan, and Amit Konar \\ Dept. of Electronics and Telecommunication Engineering, Jadavpur University, \\ Kolkata-700032, India \\ \{shreyas.sen, mail.seetharam\} @gmail.com, \\ babu25@hotmail.com
}

\begin{abstract}
This paper describes a novel scheme for automatic identification of a species from its genomic data. Random samples of a given length $(10,000$ elements) are taken from a genome sequence of a particular species. A set of 64 keywords is generated using all possible 3-tuple combinations of the 4 letters: A (for Adenine), $\mathrm{T}$ (for Thymine), $\mathrm{C}$ (for Cytosine) and $\mathrm{G}$ (for Guanine) representing the four types of nucleotide bases in a DNA strand. These $4^{3}=64$ keywords are searched in a sample of the genome sequence and their corresponding frequencies of occurrence are determined. Upon repeating this process for $\mathrm{N}$ randomly selected samples taken from the genome sequence, an $\mathrm{N} \times 64$ matrix of frequency count data is obtained. Then Principal Component Analysis is employed on this data to obtain a Feature Descriptor of reduced dimension $(1 \times 64)$. On determining the feature descriptors of different species and also by taking different samples from the same species, it is found that they are unique for a particular species while wide differences exist between those of different species. The variance of the descriptors for a given genome sequence being negligible, the proposed scheme finds extensive applications in automatic species identification.
\end{abstract}

\section{Introduction}

Genomic data mining and knowledge extraction is an interesting problem in bioinformatics. Identification of a species from its genomic database is a challenging task. The paper explores a new approach to extract genomic features of a species from its genome sequence.

Extensive works on local and global alignment of DNA sequences have already been undertaken by a number of researchers. The Smith-Waterman algorithm [1], [2] for local alignment and the Needleman-Wunsch algorithm [3] for global alignment of DNA-sequences, for instance, are two well-known algorithms for matching genome databases. These works have their own merits and limitations. The demerits include use of complicated matrix algebra and dynamic programming, and the results of sequence matching are not free from pre-calculated threshold value. It is to be noted that any of these methods stated above cannot be directly employed to identify the species from the structural signature of their genomes.

Rapid advancement in automated DNA sequencing technology [4] has generated the need for statistical summarization of large volumes of sequence data so that 
efficient and effective statistical analysis can be carried out leading to fruitful scientific results. The different known standard and popular sequence alignment algorithms and techniques for estimating the homologies [5] and mismatches among DNA sequences that are used for comparing sequences of relatively small sizes are not feasible to use when it comes to dealing with sequences having sizes varying between a few thousand base pairs to a few hundred thousand base pairs. Even for comparison of small sequences, the standard alignment and matching algorithms are known to be time consuming and laborious and the utility of more rapid and parsimonious procedures that may be somewhat rough in nature yet useful in producing quick and significant results is well appreciated. Effective analysis of large DNA sequences requires some form of statistical summarization by reducing the dimension of the data to facilitate numerical computations and at the same time to capture some of the fundamental structural information contained in the sequence data as efficiently as possible.

Traditional genetics and molecular biology have been directed towards understanding the role of a particular gene or protein in an important biological process [6]. A gene is sequenced to predict its function or to manipulate its activity or expression. This paper provides a statistical approach to handle the same problem by taking into account the genome database of a species for its identification.

The novelty of the work undertaken in the paper is summarised as follows. First, the paper takes into account frequency count of 64 three-lettered primitive DNA attributes in randomly selected samples of the genome sequences of different species like Escherichia coli (E. coli - a bacterium) [7], Drosophila melanogaster (fruit fly) [8], Saccharomyces cerevisiae (yeast) [9] and Mus musculus (mouse). Secondly, to reduce the data dimension of extracted features (here, frequency count), principal component analysis (PCA) [10] is employed on the $\mathrm{N}$ randomly selected samples of genome sequence. Thirdly, the variance of the extracted feature vectors being insignificantly small for any randomly selected input sequence, the accuracy of the results in identifying the species is very high.

The paper is divided into 5 sections. In section 2, we outline the proposed scheme for extracting the salient genomic features of a species from its genome sequence. Section 3 provides the detailed steps of using PCA in the proposed application. Diagrammatic representation of the results is presented in section 4 . The conclusions are listed in section 5 .

\section{Selection of DNA-Descriptors}

There are only 4 letters in a DNA-string; naturally the substrings could be one lettered, two lettered, three lettered or four lettered. To keep the search time optimum and moderately large search keys, we considered 3-lettered search keys only. Thus, we have $4^{3}=64$ search keys. Typical three-lettered search keys are AAA, AAC, AAG, AAT, ACA........ TTT. These 64 search keys are used to generate a $(1 \times 64)$ frequency count vector, whose each component denotes frequency of one of the 64 sub-strings or keys in a sample of the genomic data of a species.

To illustrate what we mean by frequency count, let us take the help of some examples. Consider a small portion of the sequence like ...AATCG.... It contributes a 
count of 1 each to the frequencies of occurrence of each of the 3 keywords AAT, ATC and TCG. Similarly for the substring ...TTTTT..., we get a count of 3 for the frequency of the keyword TTT. Proceeding similarly for a large sample sequence of 10,000 bases we get frequencies of all the 64 keywords in the form of a frequency count vector of dimension $(1 \times 64)$. The $(1 \times 64)$ vector is a DNA-descriptor of a given species. The DNA-descriptors are computed from different samples of a species and also for different species.

Experiments undertaken on DNA-string matching reveals that some typical substrings have a high population in the DNA-sequence of a given species. Naturally, this result can be used as a basic test criterion to determine a species from its DNAsequence.

It is important to note that the frequency counts of 64 three-element keywords in a 10,000 element string of genome sequence are more or less invariant with respect to the random sampling of the genome sequence. Naturally, our main emphasis of study was to determine whether the small difference in the counts of a given keyword in $\mathrm{N}$ samples is statistically significant. PCA provides a solution to this problem. First, the dimension of $(\mathrm{N} \times 64)$ is reduced by PCA to $(1 \times 64)$. Second, the (minor) disparity in the feature gets eliminated by PCA. Since PCA is a well-known tool for data reduction without loss of accuracy, we claim that our results on feature extraction from the genome database are also free from loss of accuracy.

\section{Application of PCA}

The methodology of employing PCA to the given problem is outlined below through the following steps:

INPUT: $\mathrm{A}$ set of $\mathrm{N}$ vectors $(1 \times 64)$ representing frequency count of 3 -tuple keywords.

OUTPUT: A minimal feature descriptor vector sufficient to describe the problem without any significant loss in data.

1. Normalization: Let the $\mathrm{i}^{\text {th }}(1 \times 64)$ input vector be $\boldsymbol{a}_{i}=\left[\begin{array}{llll}a_{i 1} & a_{i 2} & \ldots . & a_{i 64}\end{array}\right]$

To get the vector normalized we use the following transformation:

$$
a_{i k} \leftarrow a_{i k} / \sum_{j=1}^{64} a i j
$$

2. Mean adjusted data: To get the data adjusted around zero mean, we use the formula:

$$
a_{i k} \leftarrow a_{i k}-\overline{a_{i}} \quad \forall k, i
$$

where $\overline{a_{i}}=$ mean of the $\mathrm{i}^{\text {th }}$ vector

The matrix $(\mathrm{N} \times 64)$ so obtained is called the Data Adjust

$$
=\frac{1}{64} \sum_{j=1}^{64} a_{i j}=\left[a_{i j}\right]_{N \times 64}
$$


3. Evaluation of the covariance matrix: The covariance between any two vectors $a_{i}$ and $a_{j}$ is obtained by the following formula [10]:

$$
\operatorname{cov}\left(a_{i}, a_{j}\right)=c_{i j}=\frac{\sum_{k=1}^{64}\left(a_{i k}-\overline{a_{i}}\right)\left(a_{j k}-\overline{a_{j}}\right)}{(n-1)}
$$

Covariance matrix is $C=\left[c_{i j}\right]_{N \times N}$ for the $\mathrm{N}$ different $(1 \times 64)$ vectors.

4. Eigenvalue Evaluation: Computing the roots of the equation $|\mathrm{C}-\lambda \mathrm{I}|=0$, the eigenvalues of the covariance matrix $\mathrm{C}$ are obtained. There would be $\mathrm{N}$ eigenvalues of matrix $\mathrm{C}$ and corresponding to each eigenvalue there would be eigenvectors each of dimension $\mathrm{N} \times 1$.

5. Principal Component Evaluation: It is noticed that the eigenvalues have quite different values. In fact, it turns out that the eigenvector corresponding to the highest eigenvalue $\lambda_{\text {large }}$ is the Principal Component $(\mathrm{N} \times 1)$ of the data set.

$$
\therefore \text { Principal Component }=\left[p_{i}\right]_{1 \times N} \text { where } \lambda_{\text {large }} \geq \lambda_{i} \text { for } 1 \leq i \leq N
$$

6. Projection of the Data Adjust along the Principal Component: Now, to get the Feature Descriptor, the following formula is applied:

\section{Feature Descriptor $=$ Principal Component ${ }^{T} \times$ Data Adjust}

where Principal Component ${ }^{\mathrm{T}}(1 \times \mathrm{N})$ is the transpose of the Principal Component vector.

Thus we get a Feature Descriptor vector of dimension $1 \times 64$ corresponding to $\mathrm{N}$ samples of the genome sequence database of the particular species.

7. Computing the Mean Feature Descriptor: We calculate $\mathbf{M}$ such feature descriptors from different random samples and calculate a mean of these vectors and also a variance vector (both $1 \times 64$ ).

\section{Diagrammatic Representation of the Feature Descriptor}

The Feature Descriptor Diagrams for different species are described here. We could represent the Feature Descriptor using bar diagram, pie-chart or any other standard representation. However, using the polar plot we get figures which are compact yet distinct representations of the mean Feature Descriptor.

As mentioned earlier the mean Feature Descriptor is a $1 \times 64$ vector. So to construct these diagrams $360^{\circ}$ is divided into 64 equal parts, corresponding to 64 keywords. Plotting it in polar $(\mathrm{r}, \theta)$ co-ordinates with $\mathrm{r}$ as the values of the mean Feature Descriptor vector and $\Theta$ as these angles we get the Feature Descriptor diagrams. They were constructed for many species, some of which are shown below: 


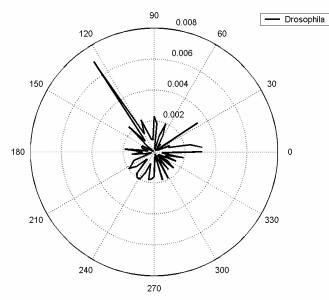

a)

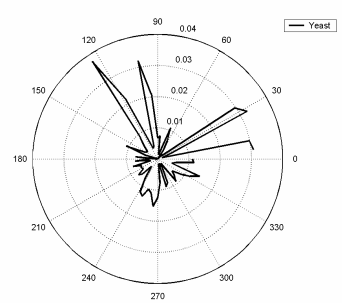

c)

Fig. 1. Feature Descriptor Diagrams for a) Drosophila, b) E. coli, c) Yeast and d) Mouse

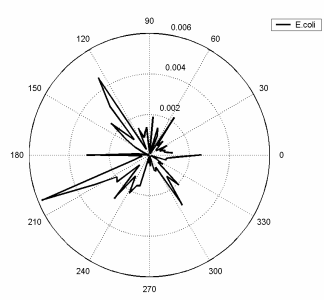

b)

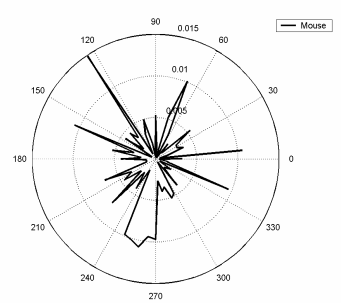

d)

Plotting the mean Feature Descriptor in polar co-ordinates gives us a figure (Feature Descriptor Diagram) for each species which we can associate with it. This differs from species to species. We have plotted below the Feature Descriptors of similar species obtained from their mitochondrial genomes.
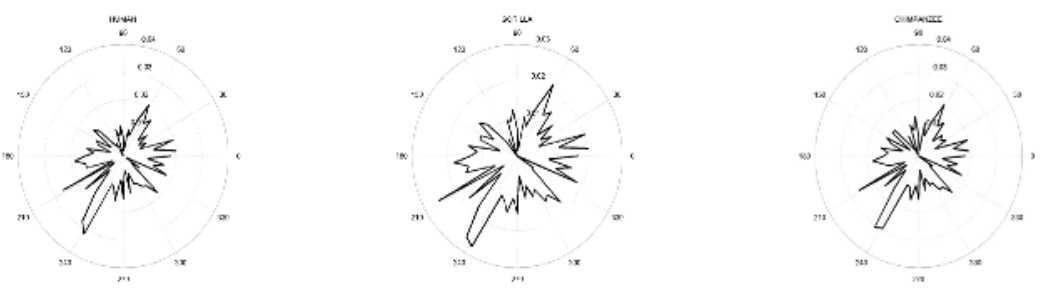

Fig. 2. Feature Descriptor Diagrams for Human, Gorilla and Chimpanzee

On observing these diagrams, we can correctly conclude that the species Human, Gorilla and Chimpanzee have many similarities in their genome characteristics which can be translated to a similarity in their biological characteristics. However it is also quite clear from these diagrams that these species have some distinctions in their genomic characteristics. So we can readily detect new species and identify known species by comparing their Feature Descriptor diagrams. Hence the Feature Descriptor Diagrams can be used as the unique representation of the genomic characteristics of the different species. 


\section{Conclusion}

This is the first work of its kind to extract information from complete genome sequences and to distinguish between species by feature descriptor diagrams.

In this process we have used PCA to reduce the large dimensions of genome sequence data without loss of accuracy. If only the frequency count is plotted then we do get some difference from species to species but it is not enough to distinguish. This is where PCA comes in. When PCA is applied to original data we get enough differences between the feature descriptor diagrams of different species which enables us to distinguish a species from the other with the help of these diagrams.

By constructing feature descriptor diagrams for several species it has been observed that they are quite different from species to species indicating that we can certainly associate each figure with each species and claim that the figure represents the species. Moreover when feature descriptor diagrams for same species are calculated they came out to be almost identical with insignificant variance.

\section{References}

1. Smith, T. F., and Waterman, M. S., "Identification of common molecular subsequences," J. Mol. Biol. pp.147, 195-197, 1981.

2. Waterman, M. S., and Eggert, M., "A new algorithm for best subsequence alignments with applications to tRNA-rRNA," J.Mol.Biol., pp. 197, 723-728, 1987.

3. Needleman, S. B., and Wunsch, C. "A general method applicable to the search for similarities in the amino sequence of two proteins." J. Mol. Biol. pp. 48,443-453, 1970.

4. Galperin, M. Y., and Koonin, E. V., Comparative Genome Analysis, In Bioinformatics- A Practical Guide to the Analysis of Genes and Proteins, Baxevins, A. D., and Oullette, B. F. F.(Eds.), Wiley-Interscience, New York, $2^{\text {nd }}$ ed., p. 387, 2001.

5. States, D. J., and Boguski, M. s., Similarity and Homology, In Sequence analysis primer Gribskov, M., and Devereux, J. (Eds.), Stockton Press, New York, pp. 92-124, 1991.

6. Mount, D. W., Bioinformatics: Sequence and Genome Analysis, Cold Spring Harbor Laboratory Press, NY, 2001.

7. Blattner, F. R., Plunkett, G., Bloch, C. A., Perna, N. T., Burland, V., Riley, M., et al., "The complete genome sequence of Escherichia coli," Vol. K-12, Science, pp. 277, 1453-1462.

8. Adams, M. D., Celniker, S. E., Holt, R. A., Evans, C. A., Gocayne, J. D., Amanatides, P. G., Scherer, S. E., Li, P. W., et al, "The genome sequence of Drosophila melanogaster," Science pp. 287, 2185-2195, 2000.

9. Cherry, J. M., Ball, C., Weng, S., Juvik, G., Schimidt, R., Alder, C., Dunn, B., Dwight, S., Riles, L. et al., "Genetic and Physical maps of Saccharomyces cerevisiae," Nature (suppl. 6632) pp. 387, 67-73, 1997.

10. Smith, L. I., "A tutorial on Principal Components Analysis,” 2002. 